\title{
Does ureteral access sheat usage lead to permanent damage in the ureter? A placebo controlled trial in a rabbit model ${ }^{1}$
}

\author{
Adnan Gücük', Gizem Söyler", Uğur Üyetürk"', Burak Yılmazv , İsmail Nalbant ${ }^{\vee}$, Sebahat Gücük ${ }^{\mathrm{VI}}$, Aysel \\ Kükner ${ }^{\mathrm{VII}}$, Ayhan Çetinkaya VIII \\ 'Associate Professor, Department of Urology, Abant Izzet Baysal University, Faculty of Medicine, Bolu, Turkey. Conception \\ and design of the study, manuscript writing, final approval. \\ "Doctor, Department of Histology and Embryology, Faculty of Medicine, Near East University, Nicosia, Cyprus. \\ Histopathological examinations. \\ I'Associate Professor, Department of Urology, Abant Izzet Baysal University, Faculty of Medicine, Bolu, Turkey. Conception \\ and design of the study. \\ "Voctor, Department of Urology, Abant Izzet Baysal University, Faculty of Medicine, Bolu, Turkey. Scientific and intellectual \\ content of the study, technical procedures. \\ ${ }^{v}$ Assistant Professor, Department of Urology, Ordu University, Faculty of Medicine, Ordu, Turkey. Scientific and intellectual \\ content of the study, technical procedures. \\ v'Assistant Professor, Department of Family Medicine, Abant Izzet Baysal University, Faculty of Medicine, Bolu, Turkey. \\ Statistical analysis, technical procedures. \\ VIIIProfessor, Department of Histology and Embryology, Faculty of Medicine, Near East University, Nicosia, Cyprus. \\ Histopathological examinations. \\ VIIIAssistant Professor, Department of Physiology, Abant Izzet Baysal University, Faculty of Medicine, Bolu, Turkey. \\ Intellectual content of the study, technical procedures.
}

\section{Abstract \\ Purpose: To evaluate the clinical stenosis or precursor histological changes that ureteral access sheaths commonly used in ureteroscopic surgeries may cause in the long term in ureter. Methods: In this study, the animals were divided into 9 groups and according to their groups, ureters of the rabbits were endoscopically fitted with $2 \mathrm{~F}$ and $3 \mathrm{~F}$ ureter catheters. The catheters were left in place and withdrawn after a specified period of time. All the ureters were excised and evaluated macroscopically, microscopically and histologically. Ureter diameters were measured and FGF-2 (+) labeled fibroblasts were counted in connective tissue as stenosis precursors.}

Results: Macroscopically or microscopically, no stenosis was found in any group. The ureter diameter of the group that were catheterized for the longest time with the catheter that had the widest diameter was significantly lower than the group with the shorter duration and the catheter with the narrower diameter and the control group. When the groups were compared in terms of their FGF values, there was a significant difference in FGF-2 counts at all three ureter levels $(p<0.05)$.

Conclusion: The use of ureteral access sheath may lead to histological changes, as its diameter and duration increase.

Key words: Ureteroscopy. Urinary Catheters. Rabbits. 


\section{Introduction}

Ureteral access sheath (UAS) is used increasing popularity among urologists as it makes it easier to enter the collective tubules in minimally invasive treatments of complex upper urinary system diseases, and minimizes the damage to the ureter causes by repeated entries and exits ${ }^{1}$. However, its disadvantages include damage to the ureter due to various mechanisms in this prevalent usage ${ }^{2}$. Various studies have shown that excessive distension of the ureters by access sheaths affects ureteral blood flow and causes ureteral ischemia in following stages $^{3}$. It was reported that the reperfusion occurring as a result of removing the access sheath may harm the ureter via free radicals ${ }^{4}$. The literature stated that access sheaths may have chronic effects in the ureter in the long-run, and recommended usage of sheaths with suitable diameters in different cases $^{3}$. To the best of our knowledge, there has been no study that investigated the effects of the duration of ischemia due to usage of sheaths and their diameter in the form that is applicable to the current clinical practice.

In the study that we planned with the reasons stated above, we placed catheters equivalent to different diameters of ureteral sheaths into rabbit ureters endoscopically for different durations. Then, we analyzed the possible permanent effects in the ureter via both visual and histopathological examination. Therefore, we aimed to describe an issue that has not been clearly explained in the literature by simulating a practice that is identical in humans on rabbits.

\section{Methods}

This study was approval from the Ethics Board for Animal Studies of Abant Izzet Baysal University.

Seventy-two healthy New Zealand White male rabbits (weight, $3.0-3.5 \mathrm{~kg}$ ) provided by the Experimental Animal Center of the University were used. The animals were housed in a temperature-controlled $(19 \mathrm{C} \pm 2 \mathrm{C})$, humidity-controlled, (40\%-70\%), and light period controlled (12h/12h light/dark cycle) environment. They were fed a standard rabbit pellet diet and had access to tap water ad libitum. While starting our study, we could not find a literature with similar endoscopic surgical procedures. Therefore, in order to familiarize with the localization of the rabbit ureter endoscopically and determine the largest diameter of ureteral sheath we can place, we calibrated the ureter via the bladder using open surgery. We determined that a maximum of a $3 \mathrm{~F}$ ureteral catheter could penetrate in rabbits of a weight of 3-3.5. As seen in Table 1 , the animals were divided into 9 groups based on the catheter diameter used and the duration. Each group contained 8 rabbits. $2 \mathrm{~F}$ catheter was applied in the first four groups and $3 \mathrm{~F}$ catheter was applied in the second four groups. After the ureteral catheter was placed, it was left there for 1, 2, 3 or 4 hours based on the groups. At the end of these durations, the catheters were removed. Only cystoscopy was applied and no catheterization was made in the 9th group which was the control group.

Table 1 - Groups according to catheter size and duration.

\begin{tabular}{llll} 
& 2F & 3F & $\begin{array}{l}\text { Cystoscopy } \\
\text { only }\end{array}$ \\
\hline 1 hour & Group 1 & Group 5 & \\
2 hours & Group 2 & Group 6 & \\
3 hours & Group 3 & Group 7 & \\
4 hours & Group 4 & Group 8 & \\
$\begin{array}{l}\text { Cystoscopy } \\
\text { only }\end{array}$ & & & Group 9 \\
(Control)
\end{tabular}

Before the interventional procedures, ketamine $\mathrm{HCl}$ at a dose of $35 \mathrm{mg} / \mathrm{kg}$ and xylazine at a dose of $10 \mathrm{mg} / \mathrm{kg}$ were administered intramuscularly for general 
anesthesia. Briefly, the animals were placed in a supine position, and their genitalia were scrubbed with povidone iodine solution. An $8 \mathrm{~F}$ pediatric Cysto-Urethroscope was used for the endoscopic operation. Based on the groups they belonged to, $2 \mathrm{~F}$ and $3 \mathrm{~F}$ ureteral catheters were placed only into the right ureters of the rabbits in correspondence to ureteral access sheaths used in humans, the catheters were left in their places for a suitable duration based on the groups, and then removed.

In order to determine the long-term effects of access sheaths, the rabbits were fed for 1 month and then sacrificed. At the end of 1 month, all ureters were excised by open surgery. In the meantime, visual evaluation was made to see whether the ureters were narrowed or whether there was an indicator of macroscopic dilatations as an indicator of such a case. They were then taken into histopathological examination for detection and comparison of issues such as long-term narrowing in the ureters in terms of the permanent effects of ischemic damage or potential pathological issues that are precursors for it. The ureters of the subject were taken and fixed in 10\% neutralized formalin. The ureters were passed through alcohol gradients and xylol by separation of upper (renal), medial and lower (bladder) pieces, and embedded in paraffin. The cross-sections with the thickness of $5 \mu \mathrm{m}$ taken from the blocks were immunohistochemically stained using hematoxylin eosin, Masson's 3-color stain and FGF-2 (rabbit polyclonal anti FGF-2, sc-7911).

In the tissue cross-sections of each subject, ureter diameters were measured with $\mathrm{x} 4$ magnification in units of micrometer from 4 different points, and averages of values were taken. In all cross-sections, the fibroblasts in the connective tissues (lamina propria) stained with FGF-2 (+) were counted under $x 40$ magnification.

The collected data were analyzed with the SPSS 20 package software. Due to the number of the units, normal distribution of the variables was tested using the Shapiro Wilk test. When the variables were not found to be normally distributed, the Kruskal Wallis-H test was utilized to analyze the differences among the groups. In the case there was significant differences in the Kruskal Wallis-H test, the groups with differences were determined using the Post-Hoc Multiple Comparisons test. While interpreting the results, the level of significance was determined as 0.05 .

\section{Results}

During the experiment, only one animal died in the 3rd postoperative day. The experiment was repeated for this animal. There was no complication seen other than this. During the removal of the ureters, the groups were evaluated both macroscopically, and microscopically for histopathological characteristics.

\section{Macroscopic}

During the excision of the ureters, all ureters were macroscopically evaluated for potential narrowing and dilatation in the proximal. However, narrowing or dilatation of the proximal as an indirect indicator of it was not seen in any groups including the groups with statistically significant differences.

\section{Microscopic}

The ureteral diameter measurements did not show narrowing in the microscopic evaluation either. In the in-group comparison of ureteral diameters, the medial and lower ureter diameters were significantly lower than the upper ureter diameter in Group 6, and the lower ureter diameter was significantly lower than the upper ureter diameter in Group 8 $(p<0.05)$. In the other groups including the control group, no significant differences were found among upper, medial and lower groups ( $p>0.05)$. 
When the upper ureter sections
were compared in the inter-group comparisons considering the average of four measurements, the diameters in the Groups 4, 6 and 8 were found significantly lower than the control group $(p<0.05)$. There were no significant differences in the medial ureter comparisons. In the comparison of the lower ureter diameters, the ureter diameter in Group 8 was found significantly lower than those in the Groups 1, 2 and 3, as well as the control group (Figure 1). Table 2 shows the inter-group comparison values in terms of ureter diameter.
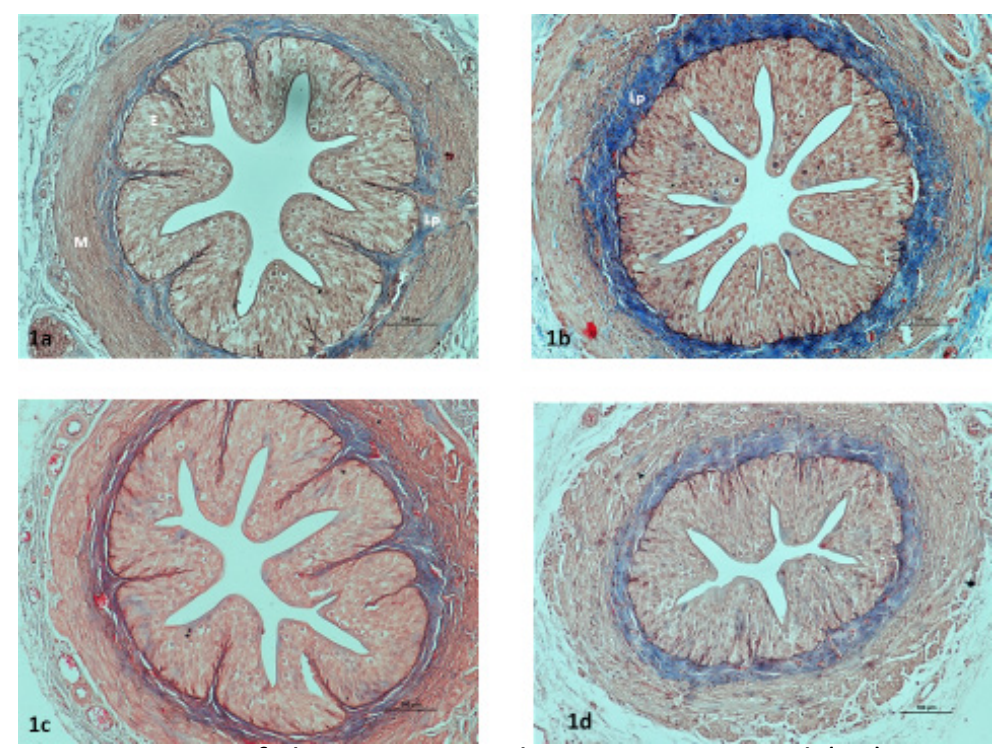

Figure 1 - Light-microscopic images of the ureters in the groups. Control (1a), Group 1 (1b), Group 2 (1c), Group 8 (1d), Epithelium (E), lamina propria (Lp), muscle layer (M). Masson's 3-color staining. Bar 100 $\mu \mathrm{m}$.

Table 2 - The results of the Kruskal Wallis $\mathrm{H}$ test regarding the difference among the groups in terms of ureter diameter (micrometer).

\begin{tabular}{llll} 
& $\begin{array}{l}\text { Upper } \\
\text { Ureter }\end{array}$ & $\begin{array}{l}\text { Medial } \\
\text { Ureter }\end{array}$ & $\begin{array}{l}\text { Lower } \\
\text { Ureter }\end{array}$ \\
\hline & Mean & & \\
Group 1 & 893.55 & 812.19 & 850.14 \\
Group 2 & 884.77 & 796.43 & 864.57 \\
Group 3 & 893.03 & 854.1 & 855.59 \\
Group 4 & 851.3 & 807.15 & 843.85 \\
Group 5 & 908.55 & 803.38 & 872.85 \\
Group 6 & 854.73 & 760.63 & 765.25 \\
Group 7 & 892.43 & 801.34 & 733.87 \\
Group 8 & 837.46 & 754.21 & 661.3 \\
Control & 1080.1 & 862.84 & 916.37 \\
H & 19.465 & 7.682 & 28.216 \\
p & $\mathbf{0 . 0 1 3}$ & 0.465 & $\mathbf{0 . 0 0 1}$ \\
\hline
\end{tabular}

No significant differences were found in the in-group comparison of upper, medial and lower sections in terms of FGF values. In the intergroup comparisons, significant differences were found in terms of FGF-2 numbers for all three levels of ureters $(p<0.05)$. These values were higher in Group 8 in the upper level in comparison to the Groups 1, 2 and 3, as well as the control group. In the medial and lower levels, these values were higher than those of the control group in the Groups 5, 6, 7 and 8 (Figure 2). Table 3 shows the data for these values. 

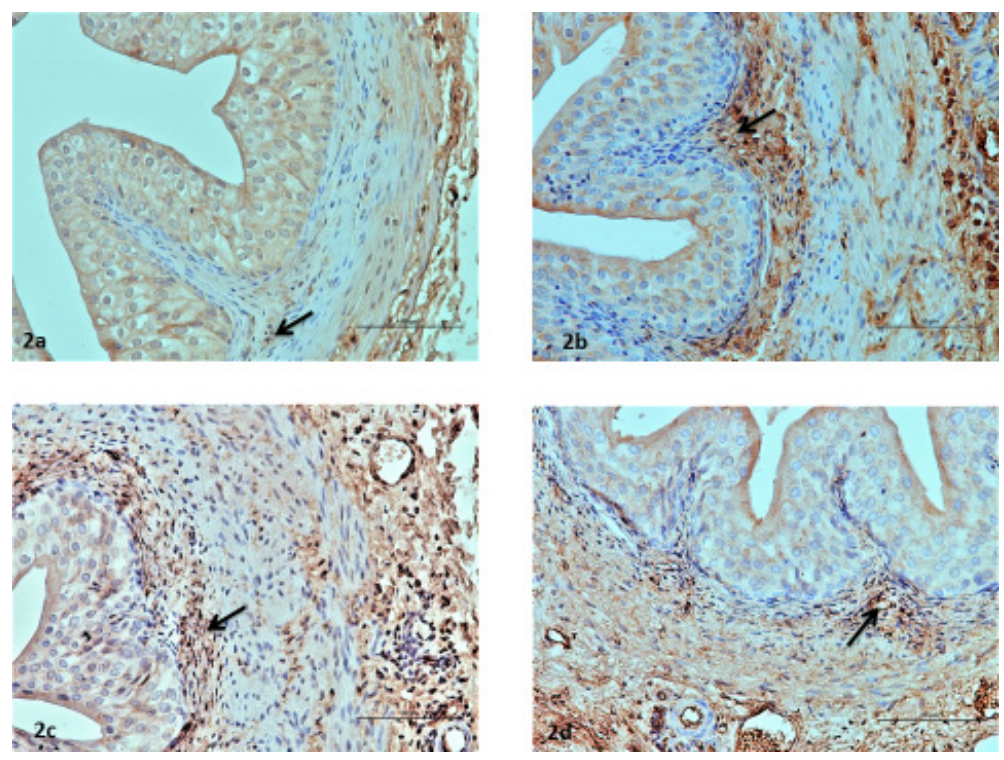

Figure 2 - Images of the FGF-2 immunohistopathologically stained ureters in the groups. Fibroblasts $(\rightarrow)$ stained positively by FGF-2 are seen in the lamina propria of the following groups: Control (2a), Group 6 (2b), Group 7 (2c), and Group 8 (2d). Bar $50 \mu \mathrm{m}$.

Table 3 - The Results of the Kruskal Wallis $\mathrm{H}$ test in terms of the differences in FGF $(+)$ values among the groups.

\begin{tabular}{clll} 
& $\begin{array}{l}\text { Upper } \\
\text { Ureter }\end{array}$ & $\begin{array}{l}\text { Medial } \\
\text { Ureter }\end{array}$ & $\begin{array}{l}\text { Lower } \\
\text { Ureter }\end{array}$ \\
\hline & Mean & & \\
Group 1 & 7 & 8.63 & 10.5 \\
Group 2 & 7.63 & 10.5 & 11.13 \\
Group 3 & 6.75 & 11.5 & 14.38 \\
Group 4 & 10.38 & 10.5 & 15.88 \\
Group 5 & 14.12 & 16.63 & 19.12 \\
Group 6 & 14.5 & 19.88 & 21.38 \\
Group 7 & 15.5 & 22.13 & 23.38 \\
Group 8 & 18.75 & 22.13 & 26.13 \\
Control & 7.63 & 7.5 & 8.25 \\
H & 37.067 & 41.529 & 42.477 \\
p & $\mathbf{0 . 0 0 1}$ & 0.001 & 0.001 \\
\hline
\end{tabular}

\section{- Discussion}

Ureteral access sheaths are routinely used today, especially in retrograd intrarenal surgeries. By mechanically dilating the ureter lumen, UAS improves imaging, makes stone removal easier, and shortens time of surgery ${ }^{3}$. It was shown that UASs increase tension in smooth muscles by stretching ureter lumen during this procedure, and they may lead to ischemia this way ${ }^{3,5}$. It is known that the higher the diameter of the UAS used, the better the image will be and the shorter the time of surgery will become. While UAS usage has various advantages over non-UAS ureteroscopy practices, there are concerns on its usage due to increased risk of ureteral damage ${ }^{6}$. Additionally, UASs may be used with an internal diameter of 9.5 French to 14 French, and an external diameter of $11.5 \mathrm{~F}$ to $18 \mathrm{~F}$. However, placing thick UASs into non-dilated ureters has potential risks. Likewise, surgery time is affected by UAS diameter in addition to factors such as stone loads, but the relationship between this time and the potential risk of damage in the ureter is not clearly known. In this study that we conducted with the purpose of determining these potential relationships, we think we partially found answers to these questions.

First of all, we macroscopically observed all ureters in the process of excising 
the animals' ureters after sacrificing them. We had aimed to detect potential narrowing and indirect indicator of it as a dilation in the proximal this way. However, we did not encounter such narrowing or dilation in any ureters. In the histological narrowing analysis, no pathology that would be reflected to the clinical results were found.

We found it suitable to make comparisons among different levels of ureters as their diameters may change on different levels. However, no statistically significant difference was found within the groups, with the exception of the Groups 6 and 8 . Theoretically, we expected that the narrowest diameters of ureters would be affected the most, and therefore, it would be more suitable to compare ureter levels with similar diameters. While the diameter difference in the control group was not significant, the finding that the medial and lower ureters in the Groups 6 and 8 were affected more, at least partially supported this expectation. Additionally, we believe that comparison of ureter diameters on different levels was useful in terms of increasing the precision of our analysis.

In the ureter diameter comparison among the groups, we found significant decreases in ureter diameters in the Groups 4, 6 and 8 . These groups represented either a long duration, or the groups treated with thicker ureter catheters. Accordingly, we found that catheter diameter and duration are parameters that may affect narrowness. Delvecciho et al. ${ }^{7}$ investigated long-term structural formation in patients treated with UAS. As a result of UAS usage, the occurrence rate of narrowness in radiological images in the first 3 months was found as $1.4 \%$. Considering that this rate was $1.2 \%$ in patients who were not treated with UAS, they concluded that UAS usage does not have an influence. Indeed, the clinical outcome of our study agreed with the findings of their study. However, considering the results in terms of the reduction in microscopic ureter diameters and similar FGF (+) outcomes in these groups, the duration and the catheter diameter affecter histological results negatively.

In the study where Lallas et $a .^{3}$ used a pig model to investigate the ischemic effects of UAS, they used $12 \mathrm{~F} / 14 \mathrm{~F}$ and $14 \mathrm{~F} / 16 \mathrm{~F}$ UASs, placed the UASs with open surgery, kept them inside for $70 \mathrm{~min}$, measured blood flow using doppler ultrasonography, and conducted a histological examination after sacrificing the animals at the end of 3 days. Histologically, they were not able to find evidence of ischemia. In difference to their study, we planned to imitate surgeries we performed on humans as much as possible. This is why we placed the ureter catheters endoscopically. We tried to determine the influential parameters by keeping the catheter diameters and durations variable among the groups. Additionally, in order to be able to measure the long-term effects of these factors in animals, we sacrificed the animals after 1 month and analyzed the ureters histologically. Lallas et $a .^{3}$ reported that UAS decreased blood flow in their sample, but the ureteral wall was left without harm due to compensatory mechanisms. Therefore, they reported that they found UAS usage to be safe. However, they stated that the chronic effects of UAS usage has not yet been cleared, and therefore, it is needed to use a suitable size of UAS in each patient. They mentioned the necessity of taking preventive measures against UAS-related damage in risky patients. In their study, the histological results reflect an earlier outcome due to the sacrifice carried out after 72 hours. On the other hand, as we carried out the sacrifice after 1 month, we analyzed later histological and microscopic outcomes. Long duration and thick catheter placement appeared as influential parameters that may lead to narrowing in terms of both ureter diameter and FGF positivity. The fact that our study did not find clinical narrowing may be related to the compensatory mechanisms mentioned above, or longer-term follow-ups 
may reveal potential narrowing.

Limitations of our study include that the process was carried out in an animal like rabbits, which have thinner ureters than those found in humans. Conducting this study on animals with similar ureter sizes, such as pigs, could have created more valid results by obtaining potentially more realistic data by using the access sheaths we use in humans. However, we did not have the means to work with this number of pigs. Additionally, while we represented UAS effects, we were not able to use real UASs as we conducted the study on rabbits. Despite all these limitations, we believe that the study simulated the outcomes of the practices we use on humans as well as possible.

\section{Conclusions}

When an upper-limit diameter such as $3 \mathrm{~F}$ is used in rabbit ureters for a long duration such as 3 or 4 hours, this causes reduction of diameter in the ureter and histological changes that may be precursors of this. Therefore, while UASs are a widely used highly useful instruments, usage time and diameter should be carefully adjusted. Usage of the shortest duration and lowest diameter of UAS may be a precaution against potential risks.

\section{References}

1. Kaplan AG, Lipkin $M E$, Scales $C D$, Preminger GM. Use of ureteral access sheaths in ureteroscopy. Nat Rev Urol. 2016;13(3):13540. doI: 10.1038/nrurol.2015.271.

2. Zelenko N, Coll D, Rosenfeld AT, Smith RC. Normal ureter size on unenhanced helical CT. Am J Roentgenol. 2004;182(4):1039-41. doI: 10.2214/ajr.182.4.1821039.

3. Lallas CD, Auge BK, Raj GV, Santa-Cruz R, Madden JF, Preminger GM. Laser Doppler flowmetric determination of ureteral blood flow after ureteral access sheath placement. J Endourol. 2002;16(8):583-90. dol: $10.1089 / 089277902320913288$.

4. Traxer O, Thomas A. Prospective evaluation and classification of ureteral wall injuries resulting from insertion of a ureteral access sheath during retrograde intrarenal surgery. J Urology. 2013;189(2):580-4. doı: 10.1016/j.juro.2012.08.197.

5. Rose JG, Gillenwater JY. Pathophysiology of ureteral obstruction. Am J Physiol. 1973;225(4):830-7. dol: 10.1152/ ajplegacy.1973.225.4.830.

6. Rizkala ER, Monga M. Controversies in ureteroscopy: wire, basket, and sheath. Indian J Urol. 2013;29(3):244-8. dol: 10.4103/0970-1591.117287.

7. Delvecchio FC, Auge BK, Brizuela RM, Weizer $A Z$, Silverstein $A D$, Lallas $C D$, Pietrow $P K$, Albala DM, Preminger GM. Assessment of stricture formation with the ureteral access sheath. Urology. 2003;61(3):518-22. PMID: 12639636.

\section{Correspondence:}

Dr. Adnan Gücük

Abant İzzet Baysal Üniversitesi Tıp Fakültesi Hastanesi

Üroloji Bölümü, Gölköy Kampusu, Bolu Turkey

Phone: +905056748193

gucukadnan@hotmail.com

Received: Jan 18, 2018

Review: Mar 20, 2018

Accepted: Apr 23, 2018
Conflict of interest: none

Financial source: Abant İzzet Baysal University Scientific Research Projects (n. 2016.08.20.1050)

${ }^{1}$ Research performed at Abant Izzet Baysal University, Experimental Animals Application and Research Center, Bolu, Turkey. 